\title{
CFD Analysis of Water Solitary Wave Reflection
}

\author{
K. Smida ${ }^{* a}$, H. Lamloumib, Z. Hafsiab and K. Maalel ${ }^{b}$ \\ ${ }^{a}$ Department of Medical Equipments Technology, Applied Medical Sciences College, Majmaah University, P.O. Box 66, Al \\ Majmaah 11952, Kingdom of Saudi Arabia \\ ${ }^{b}$ Laboratoire de Modélisation en Hydraulique et Environnement (LMHE). ENIT, B.P 37 Le Belvédère, 1002 Tunis, Tunisia
}

Received 4 April 2011; accepted 19 September 2011

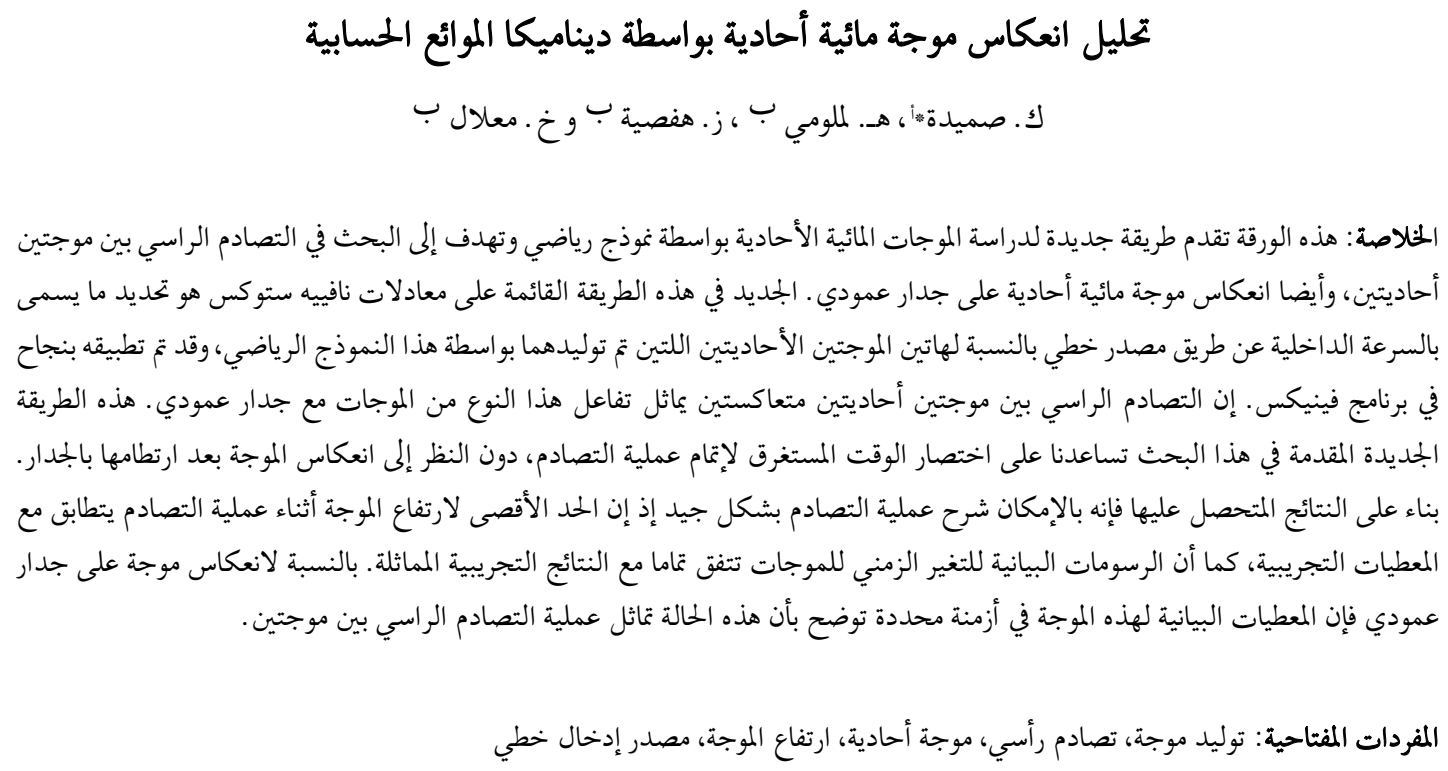

Abstract: A new numerical wave generation method is used to investigate the head-on collision of two solitary waves. The reflection at vertical wall of a solitary wave is also presented. The originality of this model, based on the Navier-Stokes equations, is the specification of an internal inlet velocity, defined as a source line within the computational domain for the generation of these non linear waves. This model was successfully implemented in the PHOENICS (Parabolic Hyperbolic Or Elliptic Numerical Integration Code Series) code. The collision of two counter-propagating solitary waves is similar to the interaction of a soliton with a vertical wall. This wave generation method allows the saving of considerable time for this collision process since the counter-propagating wave is generated directly without reflection at vertical wall. For the collision of two solitary waves, numerical results show that the run-up phenomenon can be well explained, the solution of the maximum wave run-up is almost equal to experimental measurement. The simulated wave profiles during the collision are in good agreement with experimental results. For the reflection at vertical wall, the spatial profiles of the wave at fixed instants show that this problem is equivalent to the collision process.

Keywords: Wave generation, Head-on collision, Solitary waves, Source line, Wave run-up

\section{Introduction}

The study of solitary-wave dates from 1845 when John Scott Russell (Svendssen 2006) reported his experiments. The discovery of the solitary wave have lead to many mathematical investigations that try to understand its properties. In particular, Korteweg and

$\overline{\text { *Corresponding author’s e-mail: ksmida@ksu.edu.sa }}$ de Vries derived their well-known KdV equation for water waves propagating in one direction on shallow water (Dean et al. 1991). They also found an exact solution of the $\mathrm{KdV}$ equation for a single wave propagating with a uniform velocity without changing formthe solitary wave. 
The problem of head-on collision of two solitary waves takes its origin from the study of the run-up of these waves on a vertical wall (tsunami case). The tsunamis are the most known for the several types of extreme waves (Neelamani et al. 2009) and to simulate a tsunami wave, the best and simplest model is the solitary wave. The study of the dynamics of these waves helps greatly with the protection of coastal upland from erosion which is one of the challenging problems. Different types of shore protection structures are in use around the world.

Indeed, in the absence of viscosity and in the symmetric case, both problems become equivalent. Many studies have been conducted on this problem, analytically, numerically and experimentally, including the calculation of the maximum run-up (or superlinear amplitude increase) and the phase shifts. Maxworthy (1976) conducted experiments on the collision of two solitary waves; where he showed that the maximum run-up reached a value higher than the sum of waves amplitudes before collision. Su et al. (1980) and Craig et al. (2006) presented analytical and numerical studies on the head-on collision of two solitary waves and they found that the collision was not elastic, and a small amount of energy was lost by the waves to form secondary waves. Wu (1998) presented an analytical study for head-on and following collisions of solitary waves of unequal amplitudes. He distinguished three regimes: the single peak, the double peak and the critical regime.

According to Lubin et al. (2005), there is a lack of numerical simulation models of the head-on collision waves based on Navier-Stokes equations. The most known method for linear and non linear wave generation is the internal source region proposed by Lin et al. (1999). However, the existing CFD code does not integrate any numerical wave method.

Power et al. (1984) studied the reflection of a solitary wave on a vertical wall by solving the Boussinesq equations analytically as well as numerically. The analytical solution is obtained through an asymptotic matching technique, while the numerical solution is based on a finite difference scheme. They calculated the amplitude of the maximum run-up and the time at which it reached. They found that the incident wave is not reflected immediately as predicted by linear theory. In addition, they showed that the wave undergoes a phase delay during the collision. This phase was found to be inversely proportional to the square root of the initial wave amplitude. Cooker et al. (1997) also studied the reflection of a solitary wave on a vertical wall. Their numerical approach based on a boundary integral method is used to calculate the fluid potential flow described by the Euler equations. They calculated the wall residence time, the time the wave crest remains attached to the wall, a concept introduced for the first time by (Temperville 1979). They showed that the wall residence time provides an unambiguous characterization of the wave. They compared their results on the time of attachment and detachment of the wave crest with the asymptotic formulas of ( $\mathrm{Su}$ et al. 1980). Further results on the flow were obtained, including the maximum run-up and the instantaneous forces exerted on the wall. Their numerical results on the residence time are in accordance with measurements taken from a film on the reflection of a solitary wave on a vertical wall from the experiences of (Maxworthy 1976)

The proposed numerical wave method is used in the simulation of two-dimensional head-on collision of two solitary waves and the reflection of a solitary wave at vertical wall. It is based on the Navier-Stokes equations with additional transport scalar equation to describe the free surface evolution (the Volume Of Fluid (VOF) equation). The non linear solitary waves are generated by two source lines in internal flow region. At each source line, an internal time dependent inlet velocity is imposed according to the wave characteristics (Hafsia et al. 2009). The numerical results are compared to experimental results reported by Ming et al. (2003) and non linear wave analytical solution. Numerical results given by Power et al. (1984) are used to validate the reflection of solitary wave at vertical wall.

\section{Governing Equations and Boundary Conditions}

\subsection{Transport Equations}

For unsteady flow and incompressible fluid, the mass conservation equation is written in Cartesian coordinates:

$$
\frac{\partial u}{\partial x}+\frac{\partial w}{\partial z}=0
$$

With $u$ and $w$ are the velocity components respectively in the $x$ and $z$ directions.

In order to avoid velocity damping in uniform horizontal flow, the damping force in horizontal direction is not considered. The momentum transport equation describing this velocity component is:

$$
\frac{\partial u}{\partial t}+u \frac{\partial u}{\partial x}+w \frac{\partial u}{\partial z}=-\frac{1}{\rho} \frac{\partial P}{\partial x}+v\left(\frac{\partial^{2} u}{\partial x^{2}}+\frac{\partial^{2} u}{\partial z^{2}}\right)
$$

A dissipation zone is introduced in order to damp the wave at the open boundaries. Within this region, a friction source term is added to the momentum trans- 
port equation in the vertical direction:

$\frac{\partial w}{\partial t}+u \frac{\partial w}{\partial x}+w \frac{\partial w}{\partial z}=-g-\frac{1}{\rho} \frac{\partial P}{\partial z}+v\left(\frac{\partial^{2} w}{\partial x^{2}}+\frac{\partial^{2} w}{\partial z^{2}}\right)-\gamma(x) w$

With, $P$, the pressure; $v$, kinematic viscosity; $\rho$, fluid density; $g$, gravitational acceleration and $\gamma(x)$ is a damping function equals to zero except for the added dissipation zone. A linear damping law is adopted:

$$
\gamma(x)=\alpha x+\beta
$$

\subsection{Free Surface Treatment}

The free surface displacements may be treated with a convective transport equation describing the fraction of flow (VOF) in each cell of the computation domain:

$$
\frac{\partial F}{\partial t}+\frac{\partial}{\partial x}(F u)+\frac{\partial}{\partial z}(F w)=0
$$

The free surface profile is considered as a twophase flow involving water phase and air phase. We assume that the sliding between the two phases is negligible and that there is no mass exchange across the interface. Hence, the velocity field at the free surface is continued.

\subsection{Initial and Boundary Conditions}

The initial condition considered is still water with no wave or current motion. The following boundary conditions are considered when solving the above mentioned transport equations:

* For the free surface boundary condition, the normal stress is imposed by setting the pressure $P$ equal to the atmospheric pressure $P_{\text {atm }}\left(P=P_{a t m}\right)$.

* For open boundary condition, a dissipation zone is added in order to avoid wave reflection at each end. Within such zone it is advantageous to consider, in addition to the damping friction force, a numerical dissipation by applying coarse grids in the dissipation zones. The Neumann boundary condition is specified at the end of each dissipation zone.

To generate numerically a given wave, based on the 2-D Navier-Stokes equations, the source region proposed by (Lin et al. 1999) is transformed to source line method (Fig. 1).

Since source region is reduced to a source line, the mass flux per unit time is imposed in terms of vertical velocity $w^{I}(t)$ at the bottom of the internal inlet. The mass source term in the transport equation is then considered as a time dependent inlet boundary condition.

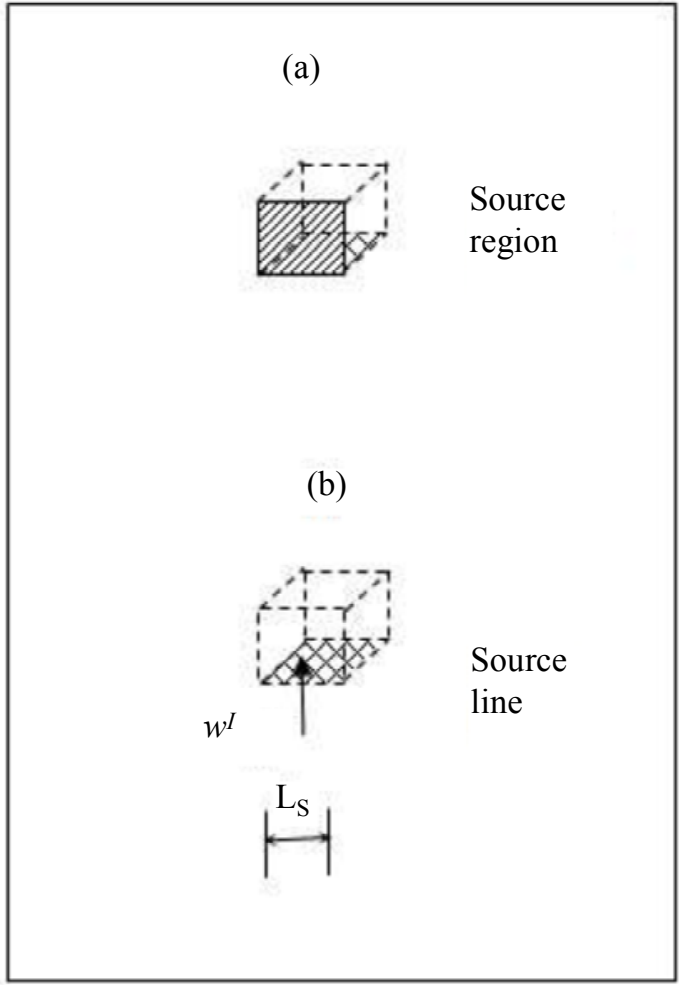

Figure 1. Internal source for wave generation; (a) The source region method proposed by (Lin et al. 1999); (b) The horizontal source line method proposed by (Hafsia et al. 2009)

\subsection{The Internal Inlet Velocity}

Following to (Hafsia et al. 2009), the wave is generated on horizontal source line with vertical pulsating velocity $w^{I}(t)$. The expression of $w^{I}(t)$ at the source line is given by the following time dependent boundary condition:

$$
w^{I}(t)=\frac{2 C \eta\left(x_{S}, t\right)}{L_{S}}
$$

Where $L_{s}$ is the length of the internal source line.

Using this transformation, the mass source region is modeled as an internal inlet imposed at the bottom of the control cell. The velocity $w^{I}(t)$ is upward or downward following that the mass is added $\left.w^{I}(t)>0\right)$ or subtracted $\left(w^{I}(t)<0\right)$.

The specified internal inlet velocity depends on the desired free surface wave profiles such as linear monochromatic wave or nonlinear solitary wave. Assuming that the source line is located at $x_{s}=0$, the free surface elevation is function only of the time $t$.

For solitary wave, we have:

$$
\eta\left(x_{s}=0, t\right)=H \operatorname{sech}^{2}\left[k\left(x_{n}-C t\right)\right]
$$


$\mathrm{H}$ : wave height; and $C$ the wave celerity given by:

$$
C=\sqrt{g d}
$$

The parameter $\mathrm{k}$ is given by:

$$
k=\sqrt{\frac{3 H}{4 d^{3}}}
$$

The wavelength of a solitary wave is theoretically infinitely long. However, for practical purposes we can define an arbitrarily wavelength as:

$$
\lambda=\frac{2 \pi}{k}
$$

The apparent wave period is defined by the following ratio:

$$
T=\frac{\lambda}{C}
$$

The distance $x_{n}$ is introduced to make the free surface elevation negligible at the initial time and is given by:

$$
x_{n}=\frac{4 d}{\sqrt{H / d}}
$$

\section{Results and Discussions}

\subsection{Introduction}

Two source lines were introduced within the computational domain to generate solitary waves propagating in constant depth (Fig. 2). Several analytical solutions are suggested in the literature for the head-on collision of two solitary waves based on Euler equations (Wu 1998).

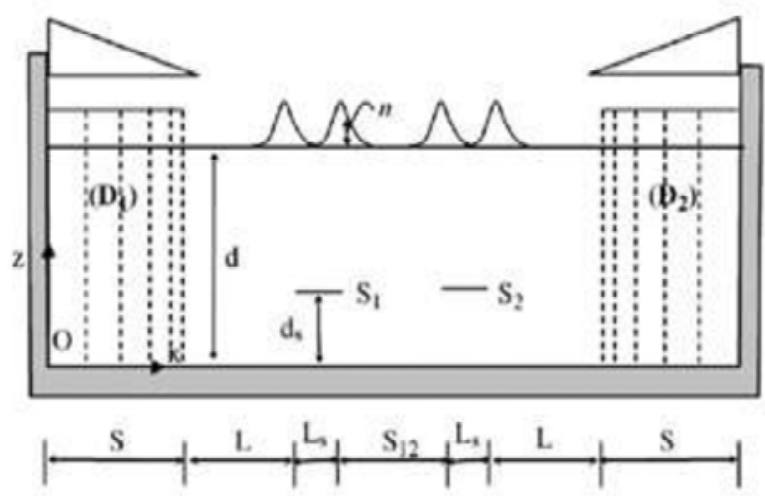

Figure 2. Computational domain and positions of the two source lines introduced to study the collision of two solitary waves at constant water depth

According to Ming et al. (2003) experiments, two solitary waves were considered with different amplitudes of $H_{1}=5.7010^{-3} \mathrm{~m}$ (left wave) and $H_{2}=5.43$
$10^{-3} \mathrm{~m}$ (right wave). The still water depth is $d=5.00$ $10^{-2} \mathrm{~m}$. The horizontal distance between the two source lines for generating these waves is $S_{12}=2.00$ $\mathrm{m}$. In horizontal direction, the left source line is located at $S_{1}=6.03 \mathrm{~m}$ and the right source at $S_{2}=8.03 \mathrm{~m}$. These two sources were located at the same elevation from the bottom $d_{s}=0.6 \mathrm{~d}$. The computational domain is discretized into uniform mesh in horizontal direction with $\Delta x=2.5010^{-2} \mathrm{~m}$. The minimum grid size in vertical direction are chosen near the free surface $\Delta z=$ $4.0310^{-4} \mathrm{~m}$. The time step is $\Delta t=1.3310^{-2} \mathrm{~s}$.

\subsection{Waves Generation}

In order to explain the solitary waves generation, Fig. 3 illustrate the free surface profiles for different times. At $t=0$ the free surface is horizontal due to that the internal inlet velocity is equal to zero. At $t=0.4 \mathrm{~s}$ (Fig. 3a), a small free surface elevation is noted. Indeed, the internal source perturbation became more influential (Figs. 3b and 3c). At $t=0.71 \mathrm{~s}$ (Fig. 3d), the two waves have remarkable shapes. After $\Delta t=$ $0.52 \mathrm{~s}$, the free surface reaches the maximum of amplitude for the two source lines, the potential energy is maximum (Fig. 3e). After this instant, the solitary waves separation can be observed (Figs. $3 \mathrm{f}$ to $3 \mathrm{~h}$ ). At $t=1.400 \mathrm{~s}$, each source line will generate two solitary waves propagating in opposite directions denoted: W1L and W1R for the source $S_{l}$ and W2L and W2R for the source $S_{2}$.

The generated four solitary waves (Fig. 4) are in accordance with the analytical solutions (Svendssen 2006).

\subsection{Head-On Collision}

A sequence of spatial profiles during collision of the two counter-propagating solitary waves is shown in Fig. 5. The free surface profiles between the times of $t=1.505 \mathrm{~s}$ and $t=1.806 \mathrm{~s}$ (Figs 5a to 5d) represent the waves propagation before collision. The numerical and experimental results are in a good agreement except some observed discrepancies. For the same head-on collision configuration, based on Euler equations, the differences between the predicted results given by (Hammack et al. 2004) and experimental data are attributed to the viscous effects and the precision of experimental measurements.

At $t=2.307 \mathrm{~s}$, the two solitary waves propagating in opposite directions (W1R and W2L) are merged into a unique wave (Fig. 5e). At this instant, the potential energy is maximum (the vertical velocity is negligible), the merging wave seems to be at rest and has a maximum amplitude higher than the sum of both incoming waves. Numerical simulation show that the 

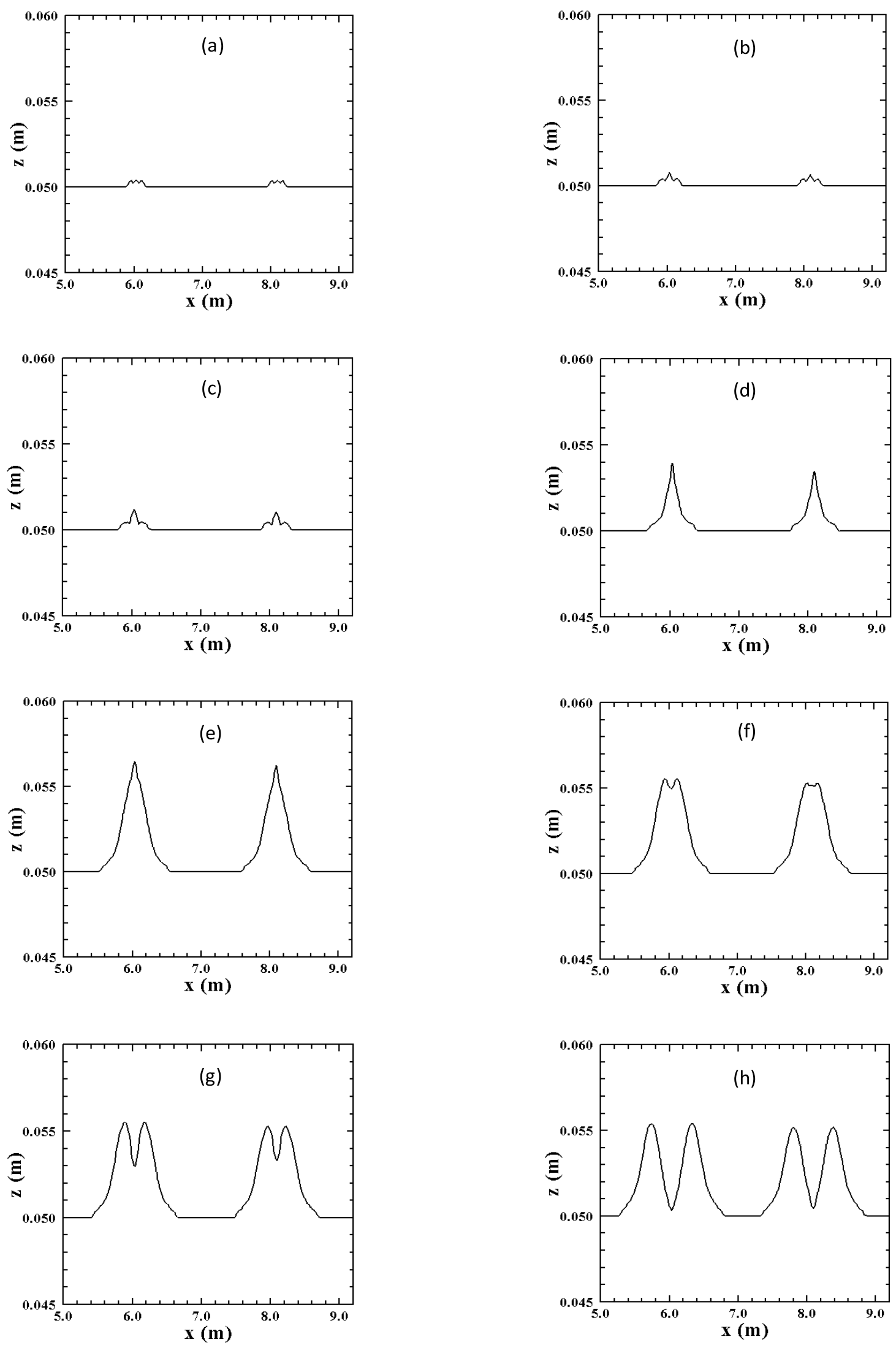

Figure 3. Free surface profiles of generated waves at different times: a) $t=0.40 \mathrm{~s}$; b) $t=0.47 \mathrm{~s}$; c) $t=$ $0.52 \mathrm{~s}$; d) $t=0.71 \mathrm{~s}$; e) $t=0.92 \mathrm{~s}$; f) $t=1.00 \mathrm{~s}$; g) $t=1.08 \mathrm{~s}$; h) $t=1.28 \mathrm{~s}$ 


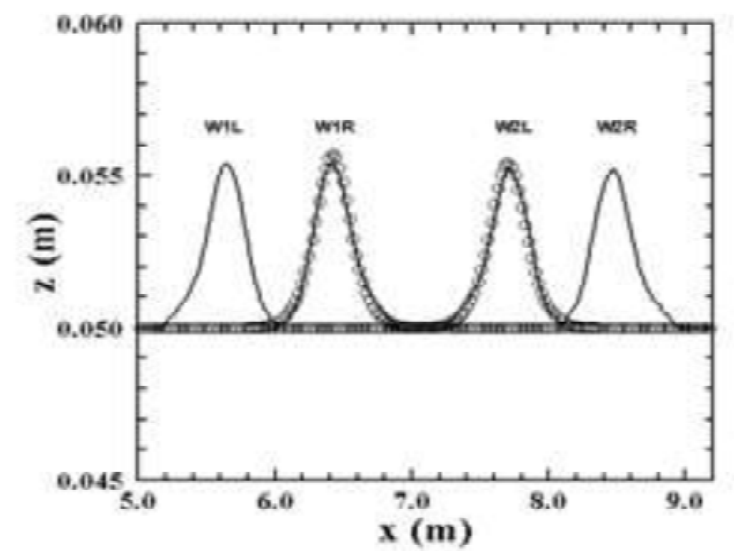

Figure 4. Comparison between analytical (ooo) and numerical free surface profiles (--) for the two solitary waves W1R and W2L before collision $(t=1.40 \mathrm{~s})$

maximum run-up $\eta_{m n}=1.0710^{-2} \mathrm{~m}$. The analytical maximum run-up $\eta_{m a}$ proposed by Su et al. (1980) is given by :

$\eta_{m a}=H_{1}+H_{2}+\frac{H_{1} H_{2}}{2}+\frac{3}{8} H_{1} H_{2}\left(H_{1}+H_{2}\right)$

This equation gives $\eta_{m a}=1.1110^{-2} \mathrm{~m}$ which is very close to $\mathrm{mn}$ given by numerical simulation. Compared to experimental results of (Ming et al. 2003), the simulated results show that the shape of the merging wave is well reproduced. Just after the time of maximum amplitude, small differences between profiles are mentioned (Fig. 5f).

After merging, at $t=3.004 \mathrm{~s}$ (Fig. $5 \mathrm{~g}$ ), both waves split apart and their initial characteristics are retrieved with a small loss of amplitude. This loss of amplitude, after collision, is consistent with the discussions of (Maxworthy 1976 and Su et al. 1980). A small phase shifts and small amount of energy loss by the crossing waves induce secondary waves (Fig. 5h) and are expected to explain this loss of amplitude. The separated waves do not undergo any numerical distortion when crossing the wave generation source lines (Hafsia et al. 2009).

\subsection{Reflection at Vertical Wall}

The problem of solitary wave reflection has attracted attention in studies of the interaction between solitary waves. Lubin et al. (2010) used the problem of solitary wave colliding a vertical end-wall to validate a numerical model simulating an unsteady two-phase tidal bore motion, in order to describe accurately the free surface behaviour and turbulent flow structure. The phenomenon of a solitary wave reflected from a vertical wall is also considered by (Lo et al. 2003) as a test case for comparison and validation of a free surface flow model. In this section, the reflection of soli-
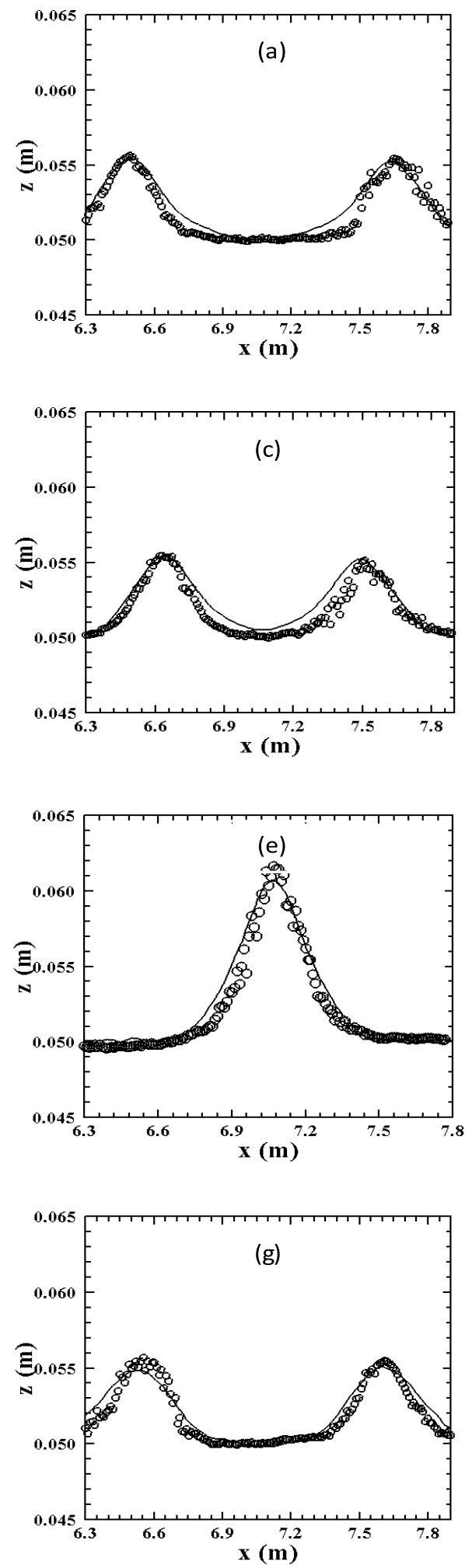

Figure 5. Comparison between experimental (ooo) during the head-on collision at different times: a) $t=1.505 \mathrm{~s}$; b) $t=1.603 \mathrm{~s}$; c) $t=1.705 \mathrm{~s}$; d) $t=1.806 \mathrm{~s} ;$ e) $t=2.307 \mathrm{~s}$ (maximum of run-up); f) $t=2.605 \mathrm{~s}$; g) $t=3.004 \mathrm{~s}$; h) $t=3.505 \mathrm{~s}$ 
tary wave at vertical wall is investigated by the source line method.

The wall is located at $x=0$, the incident solitary wave located far away the wall, is assumed to propagate in the negative $x$ direction towards the wall (Fig. $6)$.

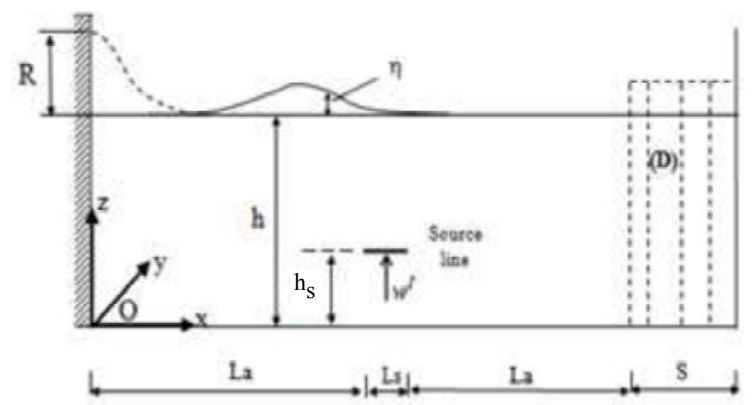

Figure 6. Computational domain and position of the source line introduced to study the reflection of a solitary wave at vertical wall

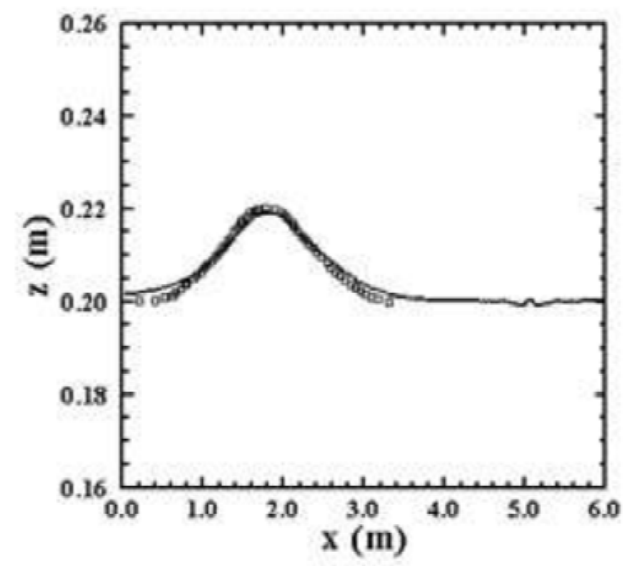

Figure 7. Comparison between numerical results given by (Power et al. 1984) (ooo) and free surface profiles $(-)$ before the reflection of a solitary wave at vertical wall; $t=4.02 \mathrm{~s}$

The solitary wave considered has an amplitude of $H=210^{-2} \mathrm{~m}$. The still water depth is $h=210^{-1} \mathrm{~m}$. In horizontal direction, the source line is located at $L a=$ $5 \mathrm{~m}$ from the vertical wall. The source was located at an elevation from the bottom $h_{s}=0.5 \mathrm{~h}$. The computational domain is discretized into uniform mesh in horizontal direction with $\Delta x=3.12510^{-2} \mathrm{~m}$. The minimum grid size in vertical direction are chosen near the free surface $\Delta z=1.110^{-3} \mathrm{~m}$. The time step is $\Delta t=$ $1.1910^{-2} \mathrm{~s}$.

Figure 8 represents the reflected wave profile at $t=6.88 \mathrm{~s}$, the reflected wave retrieve its initial shape with small loss of amplitude. In fact, after reflection the solitary wave loses energy to a dispersive wave train and loses height, so ultimately the speed of the reflected wave is smaller than before collision.

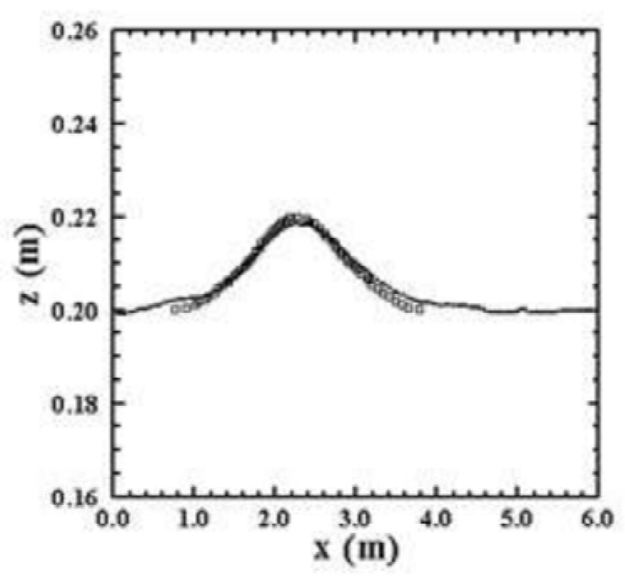

Figure 8. Comparison between numerical results given by (Power et al. 1984) (ooo) and numerical free s urface profiles $(-)$ fter the reflection of a solitary wave at vertical wall; $t=6.88 \mathrm{~s}$

Spatial profiles during reflection of the solitary wave at a vertical wall are shown in Fig. 9. From $t=4.74 \mathrm{~s}$ to $t=5.17 \mathrm{~s}$ (Figs. 9a to $9 \mathrm{~d}$ ), the wave runup due to the kinetic energy reduction is observed. Once the wave crest reaches the wall, the reflection does not occur immediately as predicted by the classical linear wave theory. The wave crest remains at the wall for a certain time, called the phase delay, to complete the reflection process. During this time delay, the wave amplitude continues to increase. The maximum run-up which is twice greater than the initial wave amplitude is reached at $t=5.31 \mathrm{~s}$ (Fig. 9e). From this instant to $t=6.02 \mathrm{~s}$ (Figs. 9f to 9j), the wave amplitude starts to decrease and the wave begins to move away from the wall and propagates in the positive $x$ direction. The numerical profiles agree fairly well with numerical free surfaces profiles given by (Power et al. 1984).

\section{Conclusions}

We have studied the head collision of two solitary waves with almost equal amplitudes by two internal mass source lines. Each source line will generate two solitary waves. The comparison with analytical non linear wave solution shows that the solitary waves are accurately generated. Between the two source lines, the two counter-propagating solitary waves are merged into a unique wave with an amplitude higher than the sum of both incoming waves. The NavierStokes solution of the maximum wave run-up is almost equal to experimental measurements. After merging, the two separated solitary waves show small differences between their initial amplitude and the simulated one. These differences are attributed to secondary waves. Since the system of two head-on colliding waves is equivalent to a single solitary wave hit- 

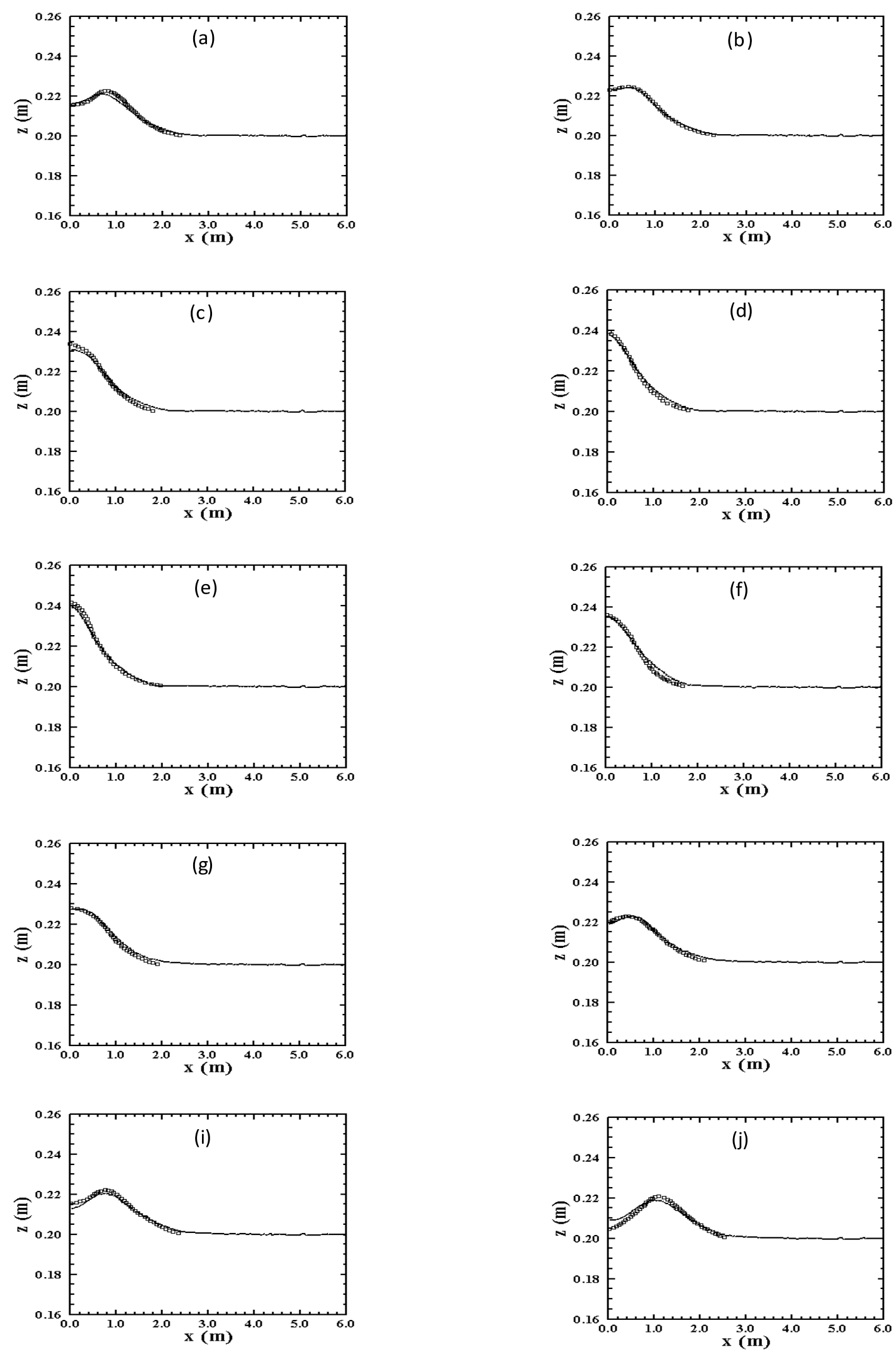

Figure 9. Comparison between numerical results given by (Power et al. 1984) (ooo) and numerical free surface profiles (-) during the reflection of a solitary wave at vertical wall at different times; a) $t=4.74 \mathrm{~s}$; b) $t=4.88 \mathrm{~s}$; c) $t=5.02 \mathrm{~s} ;$ d) $t=5.17 \mathrm{~s}$; e) $t=5.31 \mathrm{~s} ;$ f) $t=5.45 \mathrm{~s} ; \mathrm{g}) t=5.60 \mathrm{~s}$; h) $t=5.74 \mathrm{~s}$; i) $t=5.88 \mathrm{~s}$; j) $t=6.02 \mathrm{~s}$ 
ting a vertical wall, this problem is also treated in this study; using one source line for wave generation. Compared to numerical results given by (Power et al. 1984), the simulated results are considered satisfactory. As a perspective of this study, we can investigate the head-on and co-propagating collision of solitary waves with unequal amplitudes. Also the superposition of other wave types can be treated by this source line generation method.

\section{References}

Cooker MJ, Weidman PD, Bale DS (1997), Reflection of a high amplitude solitary wave at a vertical wall. J. Fluid Mech. 342:141-158.

Craig W, Guyenne P, Hammack J, Hendersen D, Sulem C (2006), Solitary wave interactions. Phys. Fluids 18:1-25.

Dean RG, Dalrymple RA (1991), Water wave for engineers and scientists. World Scientific.

Hafsia Z, Ben Haj M, Lamloumi H, Maalel K (2009), Internal inlet for wave generation and absorption treatment. J. of Coastal Engineering 56:951-959.

Hammack J, Henderson D, Guyenne P, Yi M (2004), Solitary-wave collisions. Proceedings of the $23^{\text {rd }}$ ASME Offshore Mechanics and Arctic Engineering (A symposium to honor Theodore Yao-Tsu Wu), Vancouver, Canada.

Lin P, Liu PLF (1999), Internal wave-maker for navier-stokes equations models. J. of Waterway, Port, Coastal, and Ocean Engineering 125(4): 207-215.
Lo DC, Young DL (2004), Arbitrary lagrangian-eulerian finite element analysis of free surface flow using a velocity-vorticity formulation. J. of Computational Physics 195:175-201.

Lubin P, Chanson H, Glockner S (2010), Large eddy simulation of turbulence generated by a weak breaking tidal bore. Environ Fluid Mech. 10:587602.

Lubin P, Vincent S, Caltagirone JP (2005), On the navier-stokes equations simulation of the head-on collision between two surface solitary waves. C. R. Mecanique 333:351-357.

Maxworthy T (1976), Experiments on the collisions between solitary waves. J. Fluid Mech. 76:177185.

Ming Yi, Haink Tu (2003), Head-on collision of KdV Solitons. http:math.psu.edu/dmh/Solitons/.

Neelamani S, Al-Salem K, Rakha K (2009), Extreme gravity waves in the Arabian Gulf. J. of Engineering Research 6(1):21-36.

Power H, Chwang AT (1984), On reflection of a planar solitary wave at a vertical wall. Wave Motion 6:183-195 North Holland.

Su CS, Mirie RM (1980), On head-on collisions between two solitary waves. J. Fluid Mech. 98: 509-525.

Svendsen IA (2006), Introduction to nearshore hydrodynamics. World Scientific.

Temperville A (1979), Interactions of solitary waves in shallow water theory. Arch. Mech. 31:177-184.

Wu TY (1998), Nonlinear waves and solitons in water. Physica D. 123:48-63. 